\title{
Teneurs de métaux traces dans des sols à maraîchers dans la ville d'Abidjan (Côte d'Ivoire)
}

\author{
Joseph KOUAKOU KOUASSI ${ }^{1 *}$, Edmond SIKA AHOUA ${ }^{2}$, Odette Dogbo DENEZON ${ }^{1}$ \\ Yves-Alain BEKRO ${ }^{3}$, Denis BAIZE ${ }^{4}$, Moussa BOUNAKHLA ${ }^{5}$, Fatiha ZAHRY ${ }^{5}$ et \\ Mounia TAHRI $^{5}$ \\ ${ }^{1}$ Université d'Abobo-Adjamé, Laboratoire de Biologie et Amélioration des Productions Végétales, \\ 02 BP 801 Abidjan 02, Côte d'Ivoire. \\ ${ }^{2}$ Université d'Abobo-Adjamé, Laboratoire des Sciences de l'Environnement, 02 BP 801 Abidjan 02, \\ Côte d'Ivoire. \\ ${ }^{3}$ Université d'Abobo-Adjamé, Laboratoire de Chimie Bio-Organique et de Substances Naturelles, \\ 02 BP 801 Abidjan 02, Côte d'Ivoire. \\ ${ }^{4}$ INRA Science du Sol, BP 20619, 45166 Olivet cedex, France. \\ ${ }^{5}$ Laboratoire d'Analyses Elémentaires, Centre National de l'Energie, des Sciences et des Techniques \\ Nucléaires, BP 1382 R.P. 10001 Rabat, Maroc. \\ *Auteur correspondant; E-mail: ecologue12@yahoo.fr
}

\section{RESUME}

L'objectif principal de cette étude est l'évaluation de l'état de contamination en métaux traces de sols cultivés dans la ville d'Abidjan. Pour ce faire, des sols ont été prélevés dans des fosses pédologiques sur les sites maraîchers de Marcory et Cocody et sur la ferme expérimentale de l'Université d'Abobo-Adjamé. L'analyse des échantillons a révélé que sur les 3 sites, la quasi-totalité des horizons sont sableux, acides, riches en $\mathrm{Mn}$ et $\mathrm{Fe}$. Les teneurs en $\mathrm{Cd}$ à Marcory et à Cocody, la teneur en $\mathrm{Cu}$ à Marcory, les teneurs en $\mathrm{Pb}$ à Cocody et sur la parcelle expérimentale, les teneurs en Zn sur les 3 sites, et les teneurs en Ni à Marcory et à Cocody, sont supérieures aux teneurs limites recommandées. L'acidité et la forte macroporosité des sols ont favorisé la migration verticale des métaux traces.

(C) 2012 International Formulae Group. All rights reserved.

Mots clés: métaux traces, sols à maraîchers, Abidjan, migration.

\section{INTRODUCTION}

L'épandage de déchets organiques sur les sols agricoles est considéré comme une solution simple et peu coûteuse de recyclage des éléments nutritifs (ADEME, 2003). Il en est de même de l'arrosage des cultures avec des eaux usées. Toutefois, ces pratiques agricoles peuvent poser des problèmes de qualité des sols car sources d'éléments traces métalliques (Jiries et al., 2002; Luo et al., 2003; Yadav et al., 2002). Dans le cas des sols urbains, des apports d'éléments traces sont aussi possibles par les retombées atmosphériques, les activités industrielles et domestiques (Baize et al., 2005). L'augmentation de la teneur en éléments 
traces d'origine anthropique pourrait ainsi contribuer à la contamination des plantes (Mench et Baize, 2004) ou encore à la contamination des eaux de surface et souterraines (Kouamé et al., 2006). La migration verticale des éléments traces est fonction des propriétés chimiques (nature des constituants, pH) (Bruand, 2005; Quantin et al., 2002), mais aussi physiques (perméabilité, structure) des horizons (Simpson et al., 2004). Elle est alors importante dans les sols sableux, favorisée par la macroporosité des horizons et l'absence d'éléments (matière organique, argile,...) susceptibles de retenir les métaux traces en surface des sols (Dère, 2006).

Dans la ville d'Abidjan, la production maraîchère, et plus précisément celle des légumes-feuilles, a pris une part importante dans cette activité, contribuant, ainsi, à l'approvisionnement des marchés des 10 communes en produits frais. Les sols à maraîchers sont fertilisés avec de la fiente de volaille qui, à l'instar de nombreux déchets utilisés en agriculture, renferme des matières organiques et des éléments minéraux (Doelsch, 2006) susceptibles de constituer un apport intéressant pour les sols cultivés. Mais, la présence d'éléments traces dans la fiente (Doelsch, 2006) représente une contrainte majeure à son utilisation en agriculture, car ces éléments peuvent être apportés sous des formes labiles et potentiellement biodisponibles (Tremel-Schaub et Feix, 2005). Des apports d'éléments traces aux sols à maraîchers d'Abidjan peuvent être également dûs aux engrais minéraux, aux produits phytosanitaires (Rico, 2000), aux activités domestiques et aux retombées atmosphériques.

Dans ce travail, nous avons évalué le niveau de contamination en $\mathrm{Cd}, \mathrm{Cu}, \mathrm{Pb}, \mathrm{Zn}$ et $\mathrm{Ni}$ de 5 horizons de sols à maraîchers. Ce qui nous a permis d'une part, de suivre leur migration le long des profils de sols et d'autre part, de déterminer leur origine.

\section{MATERIEL ET METHODES} Sites de prélèvement des échantillons

L'agglomération d'Abidjan est située au Sud-Est de la Côte d'Ivoire et est comprise entre les latitudes $5^{\circ} 00^{\prime}$ et $5^{\circ} 30^{\prime} \mathrm{N}$ et les longitudes $3^{\circ} 50^{\prime}$ et $4^{\circ} 10^{\prime} \mathrm{W}$. Elle s'étend sur une superficie de 57735 hectares et est composée de 10 communes avec une population estimée à 2877948 habitants (INS, 2001). Elle est en perpétuelle croissance et cette croissance est marquée par une forte urbanisation.

Sur le plan géologique, la lithologie de la ville est constituée de haut en bas de sables argileux, de sables moyens et de sables grossiers reposant sur un socle schisteux. Les formations schisteuses $\mathrm{du}$ socle sont constituées d'une part, par des roches granitiques (granite, granodiorite) et probablement des diorites quartziques (tonalites) et d'autre part, par des roches volcaniques certainement calco-alcalines (Kouamé et al., 2006).

La ville d'Abidjan possède 6 importants sites de maraîchage (A, B, C, K, $\mathrm{M}$, PB) (Figure 1) tous exposés à des pollutions d'origine urbaine et agricole. Notre étude a été menée sur les sites maraîchers de Marcory (M), de Cocody (C) et la ferme expérimentale de l'Université d'AboboAdjamé, loin de toute source de pollution agricole, qui a été nommée site témoin (T).

\section{Prélèvement des sols}

Sur chaque site d'étude, il a été délimité une parcelle d'une superficie de $60 \mathrm{~m}$ x $45 \mathrm{~m}$. Chaque parcelle a été divisée en 3 parcelles élémentaires de superficie $45 \mathrm{~m}$ x 20 $\mathrm{m}$, et une fosse pédologique, de $1 \mathrm{~m}$ de profondeur et de $1,5 \mathrm{~m}$ de longueur et de largeur, a été creusée au centre de chacune des parcelles élémentaires. Des échantillons de sol ont été prélevés selon les horizons fixes 0-20 cm, 20-40 cm, 40-60 cm, 60-80 cm, 80-100 $\mathrm{cm}$. Pour mieux évaluer l'état de contamination de l'horizon $0-20 \mathrm{~cm}$, nous 
avons prélevé 27 échantillons en différents points sur chaque parcelle. Pour les autres horizons, 3 échantillons ont été analysés. Pour chaque site, les échantillons des différents horizons restent distincts pour la phase analytique.

\section{Pré-traitement et analyse des sols}

Les échantillons de sol ont été séchés à l'étuve à $105{ }^{\circ} \mathrm{C}$ pendant $24 \mathrm{~h}$ et tamisés à 2 mm selon la norme NF X31-101 (AFNOR, 1992). Sur les échantillons de sol séchés et tamisés à $2 \mathrm{~mm}$, nous avons déterminé la granulométrie de la terre fine à trois fractions (sable, limon, argile) selon la méthode internationale de la pipette de Robinson, le $\mathrm{pH}_{\text {eau }}$ en mettant en contact $20 \mathrm{~g}$ de sol et 50 $\mathrm{mL}$ d'eau distillée avec agitation pendant $2 \mathrm{~h}$, le carbone organique selon la méthode de Walkley et Black (Mathieu et Piétain, 2003) basée sur l'oxydation de celui-ci par le dichromate de potassium $\left(\mathrm{K}_{2} \mathrm{Cr}_{2} \mathrm{O}_{7}\right)$ en milieu acide. Le dosage de l'excès de dichromate de potassium a permis de déterminer la quantité de carbone organique neutralisé.

Pour le dosage des métaux, 20 grammes de terre fine ou tamisât de sol (diamètre $<2 \mathrm{~mm}$ ) ont été broyés à $150 \mu \mathrm{m}$ et homogénéisés à l'aide d'un broyeur de marque Retsch-RM100. Pour la digestion des échantillons, $0,5 \mathrm{~g}$ de poudre fine a été dissout dans l'eau régale $\left[3 \mathrm{~mL}\right.$ de $\mathrm{HNO}_{3}$ (65\%; v/v) + $1 \mathrm{~mL}$ de $\mathrm{HCl}(37 \%$; v/v)], dans une bombe téflon d'un micro-onde de marque CEM-Mars 5, selon la norme NF X31- 415 (AFNOR, 1995). Après $16 \mathrm{~h}$ de contact, l'ensemble des bombes a été placé dans le carrousel du micro-onde pour être chauffé. Le réacteur choisi est de type XP-1500 et le programme de chauffage a été de $10 \mathrm{~min}$ à $150{ }^{\circ} \mathrm{C}, 20 \mathrm{~min}$ à $200{ }^{\circ} \mathrm{C}$ et $15 \mathrm{~min}$ à $220^{\circ} \mathrm{C}$ à 1200 Watts. Après 15 min de refroidissement, les minéralisâts ont été récupérés dans des flacons gradués, jaugés à $30 \mathrm{~mL}$ avec de l'eau distillée. Le contenu de chaque flacon a été filtré à $0,45 \mu \mathrm{m}$, et le filtrat a été acidifié avec $0,5 \mathrm{~mL}$ de $\mathrm{HNO}_{3}(65 \%$; v/v) puis conservé au réfrigérateur à $4{ }^{\circ} \mathrm{C}$ pour les analyses ultérieures. La mise en solution des métaux par l'eau régale est qualifiée de pseudo-totale (Baize et al., 2005), car le rendement de dissolution des métaux est compris entre 70 et $100 \%$ de la quantité totale (Laurent, 2003).

Le spectromètre d'absorption atomique qui a permis le dosage des métaux est de marque Varian SpectrAA-220 FS. Il est équipé d'un système de correction de bruit de fond par lampe au deutérium. Avant l'analyse des échantillons, l'appareil a été étalonné en choisissant la longueur d'onde spécifique de chaque métal. Les métaux traces $(\mathrm{Cd}, \mathrm{Cu}, \mathrm{Pb}$, $\mathrm{Zn}$, Ni) ont été analysés par atomisation électrothermique dans un four en graphite. En plus de ces 5 éléments traces, le Mn et le Fe ont été dosés dans les sols, car les oxyhydroxydes qui constituent leurs principales formes (Bataillard, 2002), sont susceptibles de retenir durablement les métaux traces (Dère, 2006). Le dosage de ces deux métaux majeurs a été effectué à la flamme. Pour chaque échantillon, 3 mesures sont faites, et la valeur retenue correspond à la moyenne.

\section{Traitement statistique des données}

Les données recueillies ont été traitées au moyen du logiciel STATISTICA 6.0. Nous avons procédé à une comparaison de moyennes en réalisant le test de Turkey à $5 \%$. Le coefficient de variation (CV) est une mesure de la dispersion relative, correspondant au rapport de l'écart type à la moyenne. Il est exprimé sous forme de pourcentage. Dans notre étude, il a été utilisé pour évaluer la variabilité ou l'homogénéité de la distribution des teneurs en métaux traces dans les profils pédologiques des sols. 


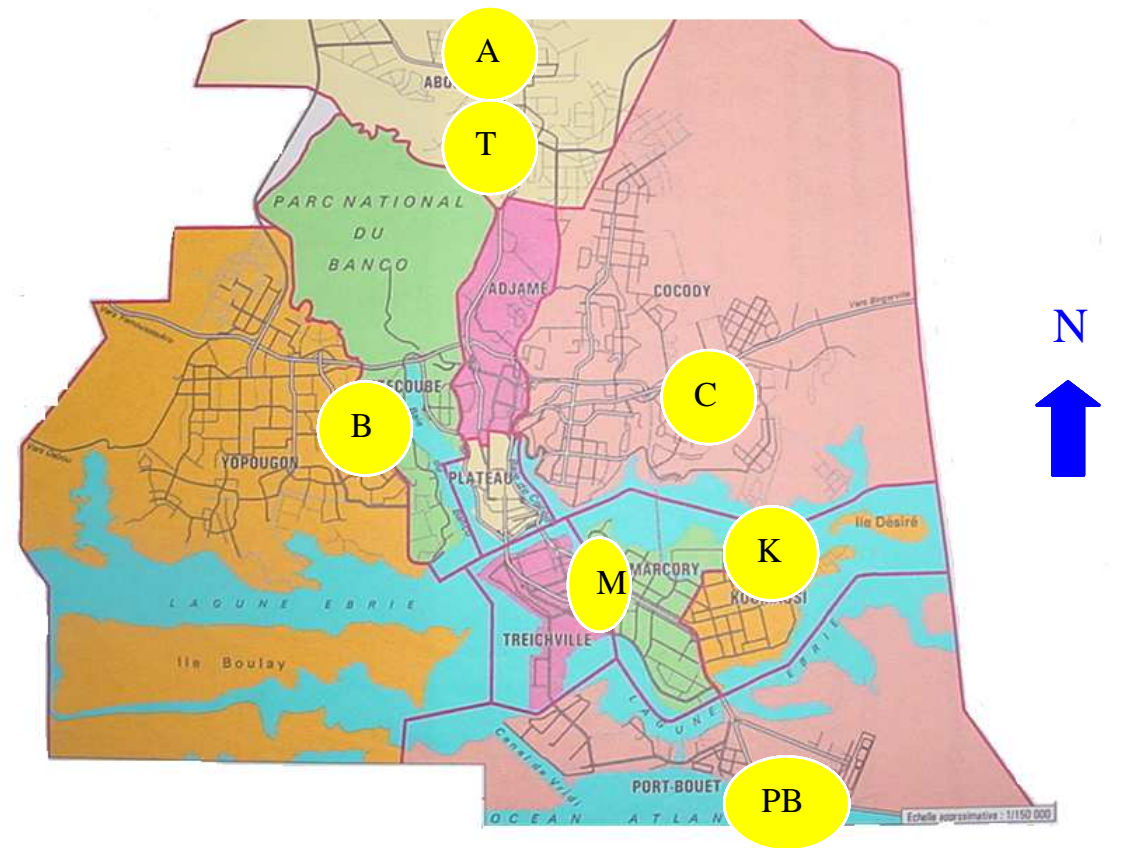

$1 / 150000$

Figure 1: Répartition des zones maraîchères au sein de l'agglomération abidjanaise.

\section{RESULTATS}

Propriétés agro-pédologiques et teneurs en Fe et en Mn des horizons des profils pédologiques des sols

Le Tableau 1 présente quelques caractéristiques agro-pédologiques et les teneurs pseudo-totales en $\mathrm{Mn}$ et en $\mathrm{Fe}$ des horizons du profil pédologique réalisé sur chaque parcelle étudiée. Hormis les horizons $20-40 \mathrm{~cm}$ et $60-80 \mathrm{~cm}$ de Cocody, qui sont, respectivement, limono-sableux et sablolimoneux, les autres horizons des sols sont sableux. À l'exception de l'horizon $80-100 \mathrm{~cm}$ de Marcory et de Cocody, les autres horizons des 3 sites sont relativement riches en matière organique (teneur en matière organique supérieure ou proche de 3\%), laquelle présente une tendance accumulatrice dans les horizons de surface. Tous les sites présentent des couches de sols acides, également riches en Fe et en Mn.

\section{Teneurs des métaux traces dans les sols}

Pour mieux apprécier les résultats sur les profils en profondeur des teneurs pseudo- totales moyennes en métaux traces, des graphiques ont été construits (Figures 2-6). En dessous de chaque graphique, nous avons présenté les différents coefficients de variation.

On note une accumulation préférentielle du Cd dans l'horizon $20-40 \mathrm{~cm}$ à Marcory, dans les horizons plus profonds $(60-80 \mathrm{~cm}$ et $80-100 \mathrm{~cm})$ à Cocody et l'horizon de surface dans le témoin. À Cocody, la teneur en $\mathrm{Cd}$ dans l'horizon de surface est du même ordre de grandeur que celles des horizons moyens $(20-40 \mathrm{~cm}$ et 40 $60 \mathrm{~cm}$ ), mais est inférieure aux teneurs obtenues dans les horizons profonds (60-80 $\mathrm{cm}$ et $80-100 \mathrm{~cm}$ ). À Marcory, le $\mathrm{Cu}$ affiche une tendance croissante de sa teneur avec la profondeur. À Cocody, le $\mathrm{Cu}$ a tendance à s'accumuler dans les 2 premiers horizons de surface; mais au-delà de ces couches, il affiche une tendance croissante de sa teneur avec la profondeur du sol. Dans le témoin, le $\mathrm{Cu}$ semble s'accumuler dans les horizons de profondeur sans tendance nette d'augmentation de sa teneur avec la profondeur du sol. À Marcory, le $\mathrm{Pb}$ présente 
une tendance décroissante de sa teneur avec la profondeur du sol. À Cocody, ce métal présente une tendance d'accumulation préférentielle dans l'horizon de surface et dans l'horizon moyen 40-60 cm. Dans le témoin, le $\mathrm{Pb}$ s'accumule préférentiellement dans les 2 derniers horizons du profil. Sur tous les sites, les teneurs en $\mathrm{Zn}$, enregistrées le long des profils de sols, ont tendance à croître et à décroître ou vis versa avec la profondeur. À Marcory, le $\mathrm{Ni}$ s'accumule préférentiellement dans les 2 premiers horizons de surface et présente des teneurs constantes dans les horizons sous-jacents. À Cocody, les teneurs en $\mathrm{Ni}$ des horizons profonds $(60-80 \mathrm{~cm}$ et $80-100 \mathrm{~cm})$ sont plus élevées que celles des horizons sus-jacents; mais la teneur du métal dans l'horizon superficiel dépasse celles des horizons moyens $(20-40 \mathrm{~cm}$ et $40-60 \mathrm{~cm})$. Dans le témoin, le Ni s'accumule préférentiellement dans l'horizon de surface et dans l'horizon moyen 40-60 cm. Il affiche une tendance décroissante de sa teneur avec la profondeur au-delà de l'horizon 40-60 cm. Il ressort de la classification des coefficients de variation mise au point par Lapointe (1992), que les coefficients qui varient entre 5,96 et $13,42 \%$, entre 17,79 et $28,32 \%$ et entre 36,36 et $97,20 \%$ expriment, respectivement, une faible variabilité (une bonne homogénéité), une variabilité moyenne (une hétérogénéité moyenne) et une forte variabilité (forte hétérogénéité) du profil pédologique pour le métal trace considéré.

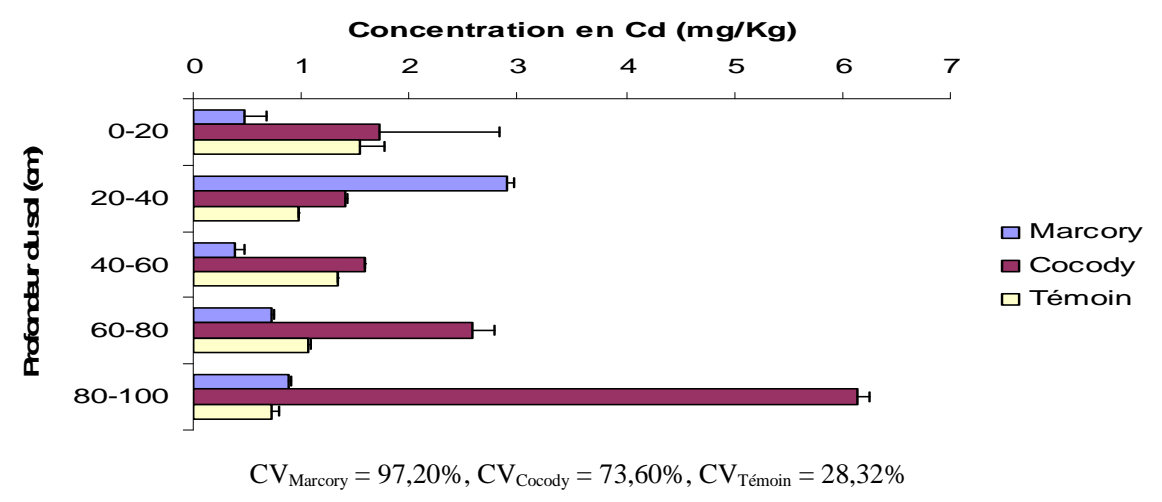

Figure 2: Profil en profondeur des teneurs pseudo-totales moyennes en $\mathrm{Cd}$.

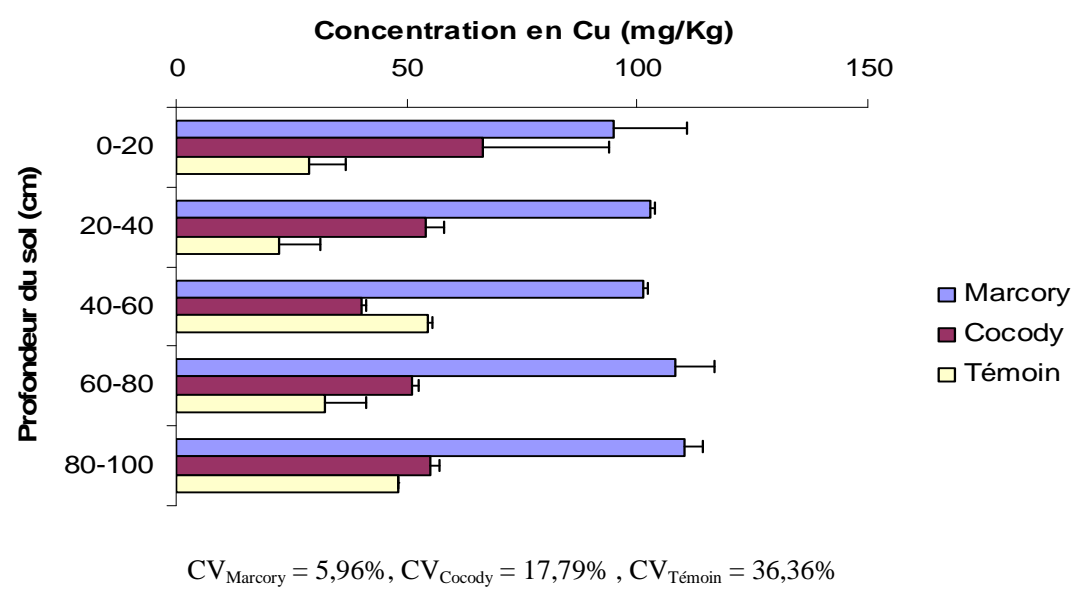

Figure 3: Profil en profondeur des teneurs pseudo-totales moyennes en $\mathrm{Cu}$. 


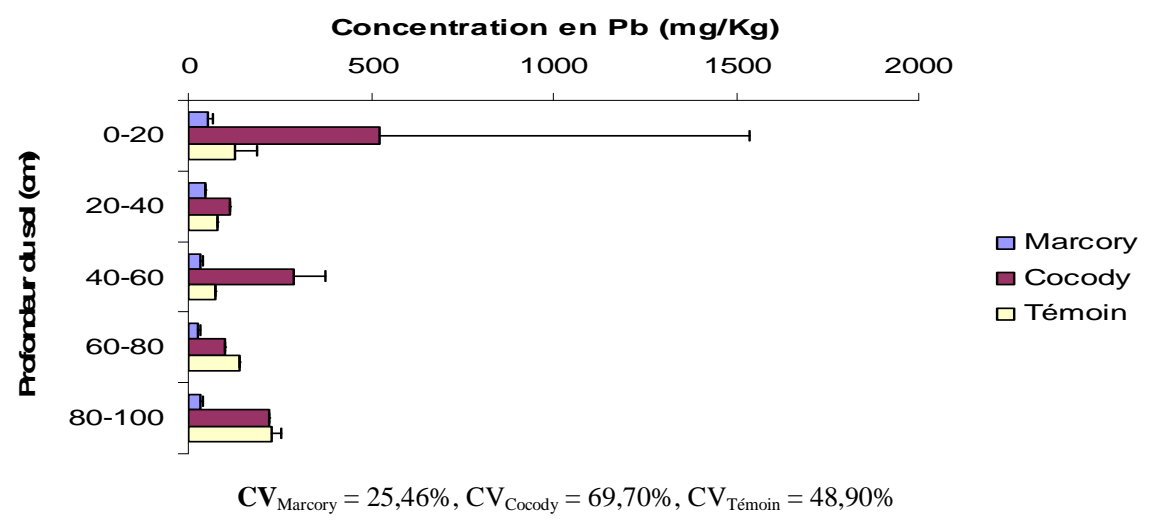

Figure 4: Profil en profondeur des teneurs pseudo-totales moyennes en $\mathrm{Pb}$.

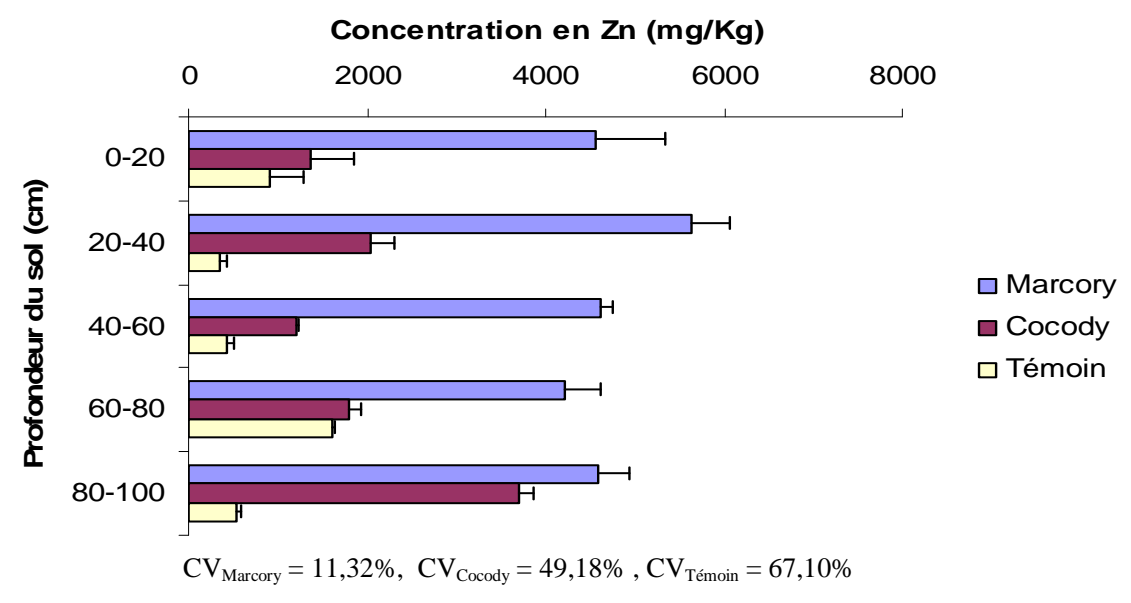

Figure 5: Profil en profondeur des teneurs pseudo-totales moyennes en Zn.

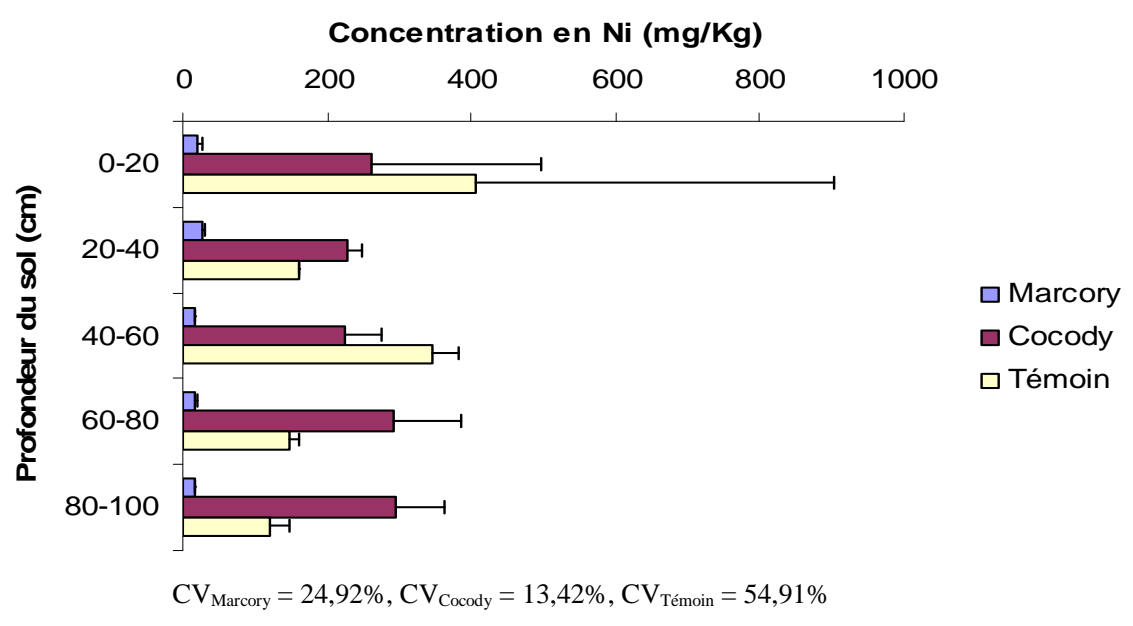

Figure 6: Profil en profondeur des teneurs pseudo-totales moyennes en Ni. 
J. KOUAKOU KOUASSI et al. / Int. J. Biol. Chem. Sci. 6(5): 2252-2262, 2012

Tableau 1: Quelques caractéristiques agro-pédologiques et teneurs pseudo-totales moyennes en Mn et en Fe des horizons des profils pédologiques.

\begin{tabular}{|c|c|c|c|c|c|c|c|c|}
\hline Sites & $\begin{array}{l}\text { Profondeur } \\
\text { du sol }(\mathrm{cm})\end{array}$ & Sable (\%) & Limon (\%) & Argile (\%) & $\mathrm{C}_{\text {org }}(\%)$ & pH & Mn (mg/kg) & $\begin{array}{c}\mathbf{F e} \\
(\mathrm{mg} / \mathrm{kg})\end{array}$ \\
\hline & $0-20(\mathrm{~N}=27)$ & $87,9 \pm 5,9^{b}$ & $7,6 \pm 3,9^{\mathrm{a}}$ & $4,5 \pm 3,2^{\mathrm{c}}$ & $5,7 \pm 3,2^{\mathrm{b}}$ & $6,4 \pm 0,6^{b}$ & $1193,2 \pm 1059^{b}$ & $5923,4 \pm 1987^{d}$ \\
\hline Marcory & $\begin{array}{c}20-40(\mathrm{~N}=3) \\
40-60(\mathrm{~N}=3) \\
60-80(\mathrm{~N}=3) \\
80-100(\mathrm{~N}=3)\end{array}$ & $\begin{array}{l}85,2 \pm 0,01^{\mathrm{a}} \\
85,3 \pm 0,01^{\mathrm{a}} \\
90,3 \pm 0,01^{\mathrm{c}} \\
89,1 \pm 0,01^{\mathrm{c}}\end{array}$ & $\begin{array}{c}13,6 \pm 0,07^{\mathrm{c}} \\
13,2 \pm 0,01^{\mathrm{c}} \\
8,6 \pm 0,02^{\mathrm{a}} \\
10,5 \pm 0,01^{\mathrm{b}}\end{array}$ & $\begin{array}{l}1,2 \pm 0,08^{\mathrm{b}} \\
1,5 \pm 0,01^{\mathrm{b}} \\
1,1 \pm 0,03^{\mathrm{b}} \\
0,4 \pm 0,02^{\mathrm{a}}\end{array}$ & $\begin{array}{l}6 \pm 5,6^{\mathrm{b}} \\
4 \pm 2,8^{\mathrm{b}} \\
5 \pm 4,3^{\mathrm{b}} \\
1 \pm 1,3^{\mathrm{a}}\end{array}$ & $\begin{array}{c}6,5 \pm 0,4^{\mathrm{b}} \\
5 \pm 1,4^{\mathrm{a}} \\
4,2 \pm 0,7^{\mathrm{a}} \\
3,8 \pm 0,3^{\mathrm{a}}\end{array}$ & $\begin{array}{c}473,8 \pm 0,7^{\mathrm{a}} \\
1350,9 \pm 3,9^{\mathrm{e}} \\
1340,4 \pm 0,8^{\mathrm{d}} \\
1327,2 \pm 19,6^{\mathrm{c}}\end{array}$ & $\begin{array}{c}5959,7 \pm 2,1^{\mathrm{e}} \\
909,8 \pm 3,8^{\mathrm{a}} \\
2130,2 \pm 2,2^{\mathrm{b}} \\
3896,1 \pm 28,9^{\mathrm{c}}\end{array}$ \\
\hline & $0-20(\mathrm{~N}=27)$ & $88,4 \pm 2,3^{\mathrm{e}}$ & $3,6 \pm 1,1^{\mathrm{a}}$ & $8 \pm 2,3^{c}$ & $3,5 \pm 1,6^{b}$ & $6,6 \pm 0,7^{\mathrm{a}}$ & $4124,5 \pm 1946^{\mathrm{e}}$ & $10007,9 \pm 3462,5^{\mathrm{c}}$ \\
\hline Cocody & $\begin{array}{c}20-40(\mathrm{~N}=3) \\
40-60(\mathrm{~N}=3) \\
60-80(\mathrm{~N}=3) \\
80-100(\mathrm{~N}=3)\end{array}$ & $\begin{array}{l}40,9 \pm 0,02^{\mathrm{a}} \\
74,9 \pm 0,01^{\mathrm{c}} \\
49,2 \pm 0,07^{\mathrm{b}} \\
84,2 \pm 0,01^{\mathrm{d}}\end{array}$ & $\begin{array}{l}56,4 \pm 0,02^{\mathrm{e}} \\
24,3 \pm 0,01^{\mathrm{c}} \\
48,8 \pm 0,17^{\mathrm{d}} \\
13,6 \pm 0,03^{\mathrm{b}}\end{array}$ & $\begin{array}{c}2,7 \pm 0,04^{\mathrm{b}} \\
0,8 \pm 0,01^{\mathrm{a}} \\
2 \pm 0,24^{\mathrm{b}} \\
2,2 \pm 0,03^{\mathrm{b}}\end{array}$ & $\begin{array}{c}4,5 \pm 0,1^{\mathrm{b}} \\
3 \pm 1,4^{\mathrm{b}} \\
3 \pm 1,4^{\mathrm{b}} \\
0,03 \pm 0,02^{\mathrm{a}}\end{array}$ & $\begin{array}{c}6 \pm 1,9^{\mathrm{a}} \\
6,2 \pm 1^{\mathrm{a}} \\
5,2 \pm 1,1^{\mathrm{a}} \\
5,3 \pm 1,3^{\mathrm{a}}\end{array}$ & $\begin{array}{c}62,9 \pm 1^{\mathrm{a}} \\
331 \pm 0,04^{\mathrm{b}} \\
941,1 \pm 1,5^{\mathrm{c}} \\
1713,6 \pm 1,4^{\mathrm{d}}\end{array}$ & $\begin{array}{l}10952 \pm 1,1^{\mathrm{d}} \\
11404,6 \pm 2^{\mathrm{e}} \\
8292,2 \pm 1,8^{\mathrm{b}} \\
4185,2 \pm 0,8^{\mathrm{a}}\end{array}$ \\
\hline & $0-20(\mathrm{~N}=27)$ & $84,4 \pm 0,9^{b}$ & $5,4 \pm 0,2^{\mathrm{a}}$ & $10,2 \pm 0,8^{\mathrm{c}}$ & $3,2 \pm 1,1^{\mathrm{a}}$ & $5,2 \pm 0,3^{\mathrm{a}}$ & $7306,7 \pm 1586^{\mathrm{c}}$ & $19985 \pm 2349,8^{\mathrm{a}}$ \\
\hline Témoin & $\begin{array}{c}20-40(\mathrm{~N}=3) \\
40-60(\mathrm{~N}=3) \\
60-80(\mathrm{~N}=3) \\
80-100(\mathrm{~N}=3)\end{array}$ & $\begin{array}{c}86 \pm 0,02^{\mathrm{c}} \\
82,7 \pm 0,04^{\mathrm{a}} \\
89,6 \pm 0,16^{\mathrm{d}} \\
85,9 \pm 0,01^{\mathrm{c}}\end{array}$ & $\begin{aligned} 12,4 & \pm 0,01^{\mathrm{c}} \\
14,4 & \pm 0,01^{\mathrm{d}} \\
9,5 & \pm 0,1^{\mathrm{b}} \\
12,8 & \pm 0,8^{\mathrm{cd}}\end{aligned}$ & $\begin{array}{l}1,6 \pm 0,01^{\mathrm{a}} \\
2,9 \pm 0,05^{\mathrm{b}} \\
0,9 \pm 0,02^{\mathrm{a}} \\
1,3 \pm 0,03^{\mathrm{a}}\end{array}$ & $\begin{array}{c}3 \pm 1,4^{\mathrm{a}} \\
2,6 \pm 0,01^{\mathrm{a}} \\
3 \pm 1,4^{\mathrm{a}} \\
4,9 \pm 1,4^{\mathrm{b}}\end{array}$ & $\begin{array}{l}4,3 \pm 0,1^{\mathrm{a}} \\
4,5 \pm 0,2^{\mathrm{a}} \\
4,3 \pm 0,01^{\mathrm{a}} \\
4,4 \pm 0,03^{\mathrm{a}}\end{array}$ & $\begin{array}{c}4456,8 \pm 53,8^{\mathrm{a}} \\
6033,4 \pm 4,1^{\mathrm{b}} \\
12012,4 \pm 2,1^{\mathrm{d}} \\
49901,5 \pm 120^{\mathrm{e}}\end{array}$ & $\begin{array}{l}28380,7 \pm 4,1^{\mathrm{b}} \\
29704,7 \pm 440^{\mathrm{c}} \\
31072,3 \pm 399^{\mathrm{d}} \\
33025,1 \pm 32,6^{\mathrm{e}}\end{array}$ \\
\hline
\end{tabular}

作

du test de Turkey au seuil de $5 \%$. 


\section{DISCUSSION}

Des phénomènes de migration verticale de la matière organique sont fréquemment observés dans les sols (Lamy, 2002; Citeau, 2004). Ce qui peut expliquer la répartition presque homogène de la matière organique le long des profils $0-80 \mathrm{~cm}$ des sols de Marcory et de Cocody. Cette migration est favorisée par la grande macroporosité des sols et les différents labours (pour ce qui concerne les sols à maraîchers) caractérisés par les différents remaniements (homogénéisation) des horizons de surface par les jardiniers. Dans le sol témoin, les teneurs en matière organique, qui augmentent faiblement avec la profondeur, indiquent que cette migration est plus importante. Les $\mathrm{pH}$, relativement plus élevés dans les horizons de surface que dans les horizons profonds, sont le reflet de l'apport de la matière organique par la fiente de volaille (pour ce qui concerne les sols à maraîchers), et de la décomposition de débris végétaux (cas du sol témoin).

Le $\mathrm{Cd}$ s'accumule préférentiellement dans les horizons riches en matière organique (Bisson et al., 2005a) et peut migrer avec le complexe organométallique (Lamy, 2002). Les profils de tous les sols sont hétérogènes par rapport à la teneur en $\mathrm{Cd}$. Les teneurs en $\mathrm{Cd}$, dans l'horizon 20-40 cm à Marcory, dans les horizons de surface à Cocody et dans le témoin, dans le fond du profil à Cocody, sont l'indice de la contribution d'une source exogène (contamination). Dans les profils pédologiques des sols des 3 sites, on note une migration $\mathrm{du} \mathrm{Cd}$, attribuable d'une part, à l'acidité des horizons et, d'autre part, à la migration du complexe organométallique.

Selon Bisson et al. (2005c), la grande partie du $\mathrm{Cu}$ reste fortement adsorbée dans les quelques centimètres supérieurs $\mathrm{du}$ sol, spécialement sur les matières organiques présentes. Par ailleurs, il migre peu en profondeur, sauf dans les conditions acides (Bisson et al., 2005c). À Marcory, la bonne homogénéité du profil pédologique en $\mathrm{Cu}$ est donc un indice de migration du $\mathrm{Cu}$ (apporté au sol par les activités anthropiques) avec la matière organique. À Cocody, les teneurs en
$\mathrm{Cu}$, plus élevées dans les 2 premiers horizons du sol que dans les horizons de profondeur, et la faible hétérogénéité du profil, sont une indication d'une faible contribution d'une source exogène (anthropique). Le $\mathrm{Cu} \mathrm{a}$, probablement, faiblement migré avec la matière organique. Dans le témoin, les teneurs en $\mathrm{Cu}$ dans les 3 derniers horizons sont aussi l'indice d'une migration du $\mathrm{Cu}$. Les profils pédologiques des sols de tous les sites sont hétérogènes pour leur teneur en $\mathrm{Pb}$. Le $\mathrm{Pb}$ présente une forte affinité pour la matière organique et l'argile (Bisson et al., 2003). Les teneurs en $\mathrm{Pb}$, dans les 2 premiers horizons de surface à Marcory, dans les horizons $0-20 \mathrm{~cm}$ et 40-60 $\mathrm{cm}$ à Cocody, dans l'horizon de surface et dans les 2 derniers horizons de profondeur du témoin, indiquent la contribution d'une contamination. À Cocody, le $\mathrm{Pb}$, à l'instar de nombreux éléments traces, a, probablement, migré (Dère, 2006) avec le complexe organométallique. Dans le profil pédologique du sol témoin, le $\mathrm{Pb}$ a également migré. Les activités agricoles (surtout l'épandage de la fiente de volaille) ont contribué à la contamination des sols à maraîchers en $\mathrm{Zn}$. La forte teneur en $\mathrm{Zn}$ dans l'horizon de surface du sol témoin indique aussi une contamination. Le $\mathrm{Zn}$ étant reconnu pour sa forte mobilité dans les sols acides (Citeau, 2004), son comportement le long des profils pédologiques des sols des 3 sites est celui d'une migration. Selon Bisson et al. (2005b), le gradient de ce métal qui tend à diminuer puis à croître, ou vis versa, avec la profondeur, est le fait des oxyhydroxydes de $\mathrm{Fe}$ et de Mn qui visent à retarder sa mobilité. À Cocody, l'horizon $20-40 \mathrm{~cm}$, de par sa texture limono-sableuse, se comporte comme une barrière à la mobilité du $\mathrm{Zn}$.

À Marcory, le Ni apporté au sol par les activités anthropiques migre et a tendance à s'accumuler dans l'horizon moyen $20-40 \mathrm{~cm}$ (relativement plus riche en matière organique que les autres couches) comme le Cd. Cette migration est attribuée aux $\mathrm{pH}$ acides des couches de sol (Bisson et al., 2006). Le profil de sol à Cocody apparât homogène pour ses teneurs en $\mathrm{Ni}$, mais la concentration en $\mathrm{Ni}$, 
plus élevée dans l'horizon de surface que dans les horizons moyens $(20-40 \mathrm{~cm}$ et $40-60 \mathrm{~cm})$, est la preuve que des apports de $\mathrm{Ni}$ sont dûs aux activités anthropiques. Le comportement du métal dans le profil pédologique est celui d'une migration. Dans le témoin, l'hétérogénéité du profil de sol pour ses teneurs en $\mathrm{Ni}$, l'accumulation préférentielle du métal dans l'horizon de surface et les teneurs en $\mathrm{Ni}$, qui tendent à décroître avec la profondeur à partir de l'horizon 40-60 cm, sont l'indice de la contribution d'une source exogène. La teneur en $\mathrm{Ni}$ enregistrée dans l'horizon 40-60 cm traduit une migration, et cette mobilité du métal a dû être favorisée par l'acidité des couches de sol.

La contamination en métaux traces des sols à maraîchers est attribuable aux pratiques agricoles, aux retombées atmosphériques et aux eaux de ruissellement, tandis que celle de la parcelle témoin est attribuable aux retombées atmosphériques et/ou à des activités anthropiques antérieures (activités domestiques et pratiques agricoles).

Nos résultats sur la migration des métaux traces sont similaires à ceux de Dère (2006) qui a également noté une migration du $\mathrm{Cd}$, du $\mathrm{Cu}$, du $\mathrm{Zn}$ et du $\mathrm{Ni}$ dans les sols sableux, faiblement alcalins, de Pierrelaye dans la banlieue parisienne. Mais, nos résultats sont différents de ceux obtenus par Kouamé et al. (2006) sur la décharge d'Akouédo à Abidjan. En effet, ces auteurs ont noté une rétention du $\mathrm{Cd}$, du $\mathrm{Cu}, \mathrm{du} \mathrm{Pb}$ et du Zn dans les couches superficielles des sols; cette immobilisation des métaux a été attribuée à la richesse de ces couches en matière organique et à leur $\mathrm{pH}$ alcalin.

Les teneurs pseudo-totales moyennes en métaux traces enregistrées dans les profils pédologiques des sols des 3 sites étudiés ont été comparées aux valeurs limites des teneurs totales en éléments traces $(2 \mathrm{mg} / \mathrm{kg}$ en $\mathrm{Cd}, 100$ $\mathrm{mg} / \mathrm{kg}$ en $\mathrm{Cu}$ et en $\mathrm{Pb}, 300 \mathrm{mg} / \mathrm{kg}$ en $\mathrm{Zn}, 50$ $\mathrm{mg} / \mathrm{kg}$ en $\mathrm{Ni}$ ) de la réglementation française relative à l'épandage de boues de stations d'épuration urbaine sur les sols agricoles (Arrêté du 8 janvier, 1998). Il ressort de cette comparaison que les teneurs en Cd à Marcory et à Cocody, la teneur en $\mathrm{Cu}$ à Marcory, les teneurs en $\mathrm{Pb}$ à Cocody et dans le témoin, les teneurs en $\mathrm{Zn}$ sur les 3 sites, les teneurs en $\mathrm{Ni}$ à Marcory et à Cocody, sont supérieures aux teneurs limites recommandées.

\section{Conclusion}

L'évolution des métaux traces le long du profil du sol présente une variabilité selon le type de parcelle et selon le métal trace considéré. La mobilité de la matière organique, liée à la structure particulaire des horizons de sol, et l'acidité de ces horizons de sol, ont favorisé la migration verticale des métaux traces.

Des teneurs anomaliques en métaux traces ont été enregistrées aussi bien dans les sols à maraîchers que dans le sol témoin. Ces teneurs mesurées dans les sols résultent de la composition chimique initiale héritée du matériau géologique dont les sols sont issus (concentration pédo-géochimique naturelle) et des apports anthropiques. L'absence de données sur les fonds pédo-géochimiques naturels ne nous a donc pas permis d'estimer la quantité de métaux traces apportés par chaque source. C'est pourquoi, les futurs travaux porteront sur les teneurs en métaux traces de la roche mère qui a donné naissance aux différents sols.

\section{REMERCIEMENTS}

Les auteurs remercient l'UNESCO, la Fondation Internationale pour la Science (FIS) et l'Organisation d'Interdiction des Armes Chimiques (OIAC) qui ont financé cette étude.

\section{REFERENCES}

ADEME. 2003. Teneurs des plantes à vocation alimentaire en éléments traces suite à l'épandage de déchets organiques, synthèse d'essais agronomiques français et modélisation des transferts sol-plante. ADEME, 75 p.

AFNOR. 1992. Norme française. AFNOR NF X 31-101. Qualité des sols. Préparation d'un échantillon de sol pour analyses physico-chimiques, AFNOR, 15-21. 
AFNOR. 1995. Norme française. AFNOR NF X31-415. Qualité des sols. Extraction des éléments en traces solubles dans l'eau régale, AFNOR, 455-463.

Arrêté du 8 janvier 1998 modifiant le décret N97-1133 du 8 décembre 1997 relatif à l'épandage des boues issues du traitement des eaux usées. Journal Officiel de la République française, 1563-1571.

Baize D, Sterckeman T, Piquet A, Ciesielski H, Béraud J, Bispo A. 2005. Dérogations relatives à la réglementation sur l'épandage des boues de stations d'épuration. Comment formuler une demande pour les sols à teneurs naturelles élevées en éléments traces métalliques? $145 \mathrm{p}$.

Bataillard P. 2002. Evolution de la spéciation du plomb et du cadmium dans les sols. Thèse de Doctorat, Ecole Nationale du Génie Rural des Eaux et des Forêts, 251p.

Bisson M, Hulot C, Lacroix G, Lefèvre JP, Magaud H, Oberson D, Geneste R, Morin A, Pépin G. 2003. Fiche de données toxicologiques et environnementales des substances chimiques. Plomb et ses dérivées, $90 \mathrm{p}$.

Bisson M, Diderich R, Houeix N, Hulot C, Lacroix G, Lefèbre JP, Leveque $S$, Magaud H, Morin A, Pepin G, Pichard A. 2005a. Fiche de données toxicologiques et environnementales des substances chimiques. Cadmium et ses dérivées, 60 p.

Bisson M, Diderich R, Hulot C, Houeix N, Lacroix G, Lefèvre JP, Leveque S, Magaud H, Morin A. 2005b. Fiche de données toxicologiques et environnementales des substances chimiques. Zinc et ses dérivées, 69 p.

Bisson M, Houeix N, Gay G, Jolibois B, Lacroix G, Hulot C, Lefèvre JP, Magaud H, Morin A, Tissot S. 2006. Fiche de données toxicologiques et environnementales des substances chimiques. Nickel et ses dérivées, 71 p.

Bisson M, Houeix N, Gay G, Lacroix G, Lefèbre JP, Magaud H, Migne V, Morin
A, Tissot S. 2005c. Fiche de données toxicologiques et environnementales des substances chimiques. Cuivre et ses dérivées, $66 \mathrm{p}$.

Bruand A. 2005. Toward conditions favourable to mobility of trace elements in soils. C. R. Geoscience, 337: 549-550.

Citeau L. 2004. Etude des colloïdes naturels présents dans les eaux gravitaires de sols contaminés : relation entre nature des colloïdes et réactivité vis-à-vis des métaux $(\mathrm{Zn}, \mathrm{Cd}, \mathrm{Pb}, \mathrm{Cu})$. Thèse de Doctorat, Institut National d'Agronomie Paris-Grignon, 251p.

Dère C. 2006. Mobilité et redistribution à long terme des éléments traces métalliques exogènes dans les sols. Application à des Luvisols pollués par 100 ans d'épandage d'eaux usées brutes dans la plaine de Pierrelaye. Thèse de Doctorat, Institut National d'Agronomie Paris-Grignon, $106 \mathrm{p}$.

Doelsch E. 2006. Evaluation de la biodisponibilité des éléments traces pour les cultures maraîchères. CIRAD, Chambre d'Agriculture de la Réunion, 42 p.

INS. 2001. Recensement Général de la Population et de l'Habitation (RGPH) 1998. Données socio-démographiques et économiques des localités, résultats définitifs par localités, région des lagunes, vol.III, tome 1 . INS, $43 \mathrm{p}$.

Jiries AG, Al-Nasir FM, Beese F. 2002. Pesticide and heavy metals residue in wastewater, soil and plants in wastewater disposal site near Al-Lajoun Valley, Karak/Jordan. Water Air Soil Pollut., 133(1-4): 97-104.

Kouamé KI, Gone DL, Savane I, Kouassi EA, Koffi K, Goula BT, Diallo M. 2006. Mobilité relative des métaux lourds issus de la décharge d'Akouédo et risque de contamination de la nappe du Continental Terminal (Abidjan-Côte d'Ivoire). Afrique Sciences, 2(1): 39-56.

Lamy I. 2002. Réactivité des matières organiques des sols vis-à vis des métaux : cas des matières organiques particulaires 
et des matières organiques solubles. $7^{\text {ièmes }}$ journées nationales de l'etude des sols, orléans (France). Association Française pour l'Etude des Sols, 51-52.

Lapointe JJ. 1992. La Conduite d'une Etude de Besoins en Education et en Formation: une Approche Systémique. Presse de 1'Université du Québec: Sillery, Québec; p. 256.

Laurent C. 2003. Les modèles d'adsorption des métaux en traces $(\mathrm{Zn}, \mathrm{Cd}, \mathrm{Cu}$ et $\mathrm{Pb})$ dans les sols: synthèse bibliographique et validation. Mémoire de stage de Diplôme d'Etudes en Pollution Chimique et Environnement, Université Paris XI, Orsay, $55 \mathrm{p}$.

Luo Y, Jiang X, Wu L, Song J, Wu S, Lu R. 2003. Accumulation and chemical fractionation of $\mathrm{Cu}$ in a paddy soil irrigated with $\mathrm{Cu}$-rich wastewater. Geoderma, 115(1-2): 113-120.

Mathieu C, Pietain F. 2003. Analyse Chimique des Sols: Méthodes Choisies. Edition Tec \& Doc: France; 387 p.

Mench M, Baize D. 2004. Contamination des sols et de nos aliments d'origine végétale par les éléments en traces, mesures pour réduire l'exposition. Courrier de l'environnement de l'INRA, 52: 31-56.

Quantin C, Becquer T, Berthelin J. 2002. Mnoxide: a major source of easily mobilisable $\mathrm{Co}$ and $\mathrm{Ni}$ under reducing conditions in New Caledonia Ferralsols. C. R. Geoscience, 334(4): 273-278.

Rico A. 2000. Pollutions et pratiques agricoles. Deux concepts: dose journalière admissible et chimiodéfense. C.R. Acad. Sci. Paris, Sciences de la Vie, 323: 435-440.

Simpson SL, Maher EJ, Jolley DF. 2004. Processes controlling metal transport and retention as metal-contaminated groundwaters efflux through estuarine sediments. Chemosphere, 56(9): 821-831.

Tremel-Schaub A, Feix I. 2005. Contamination des Sols: Transferts des Sols Vers les Plantes. EDP Sciences, ADEME; $416 \mathrm{p}$.

Yadav RK, Goyal B, Sharma RK, Dubey SK, Minhas PS. 2002. Post-irrigation impact of domestic sewage effluent on composition of soils, crops and ground water- a case study. Environ. Int., 28(6): 482-486. 